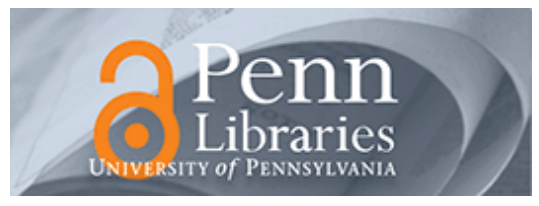

University of Pennsylvania

ScholarlyCommons

$9-1-1985$

\title{
Spin-Wave Excitations and Perpendicular Susceptibility of a Diluted Antiferromagnet Near Percolation Threshold
}

Deepak Kumar

A. Brooks Harris

University of Pennsylvania, harris@sas.upenn.edu

Follow this and additional works at: https://repository.upenn.edu/physics_papers

Part of the Physics Commons

\section{Recommended Citation}

Kumar, D., \& Harris, A. (1985). Spin-Wave Excitations and Perpendicular Susceptibility of a Diluted Antiferromagnet Near Percolation Threshold. Physical Review B, 32 (5), 3251-3262. http://dx.doi.org/ 10.1103/PhysRevB.32.3251

This paper is posted at ScholarlyCommons. https://repository.upenn.edu/physics_papers/384

For more information, please contact repository@pobox.upenn.edu. 


\title{
Spin-Wave Excitations and Perpendicular Susceptibility of a Diluted Antiferromagnet Near Percolation Threshold
}

\author{
Abstract \\ The long-wavelength excitations of a diluted antiferromagnet near the percolation threshold $\mathrm{p}_{\mathrm{c}}$ are \\ studied. Within the hydrodynamic theory, the excitation frequency depends on two parameters, $A$ and $\chi_{\mathrm{t}} . A$ \\ is the stiffness associated with the spatial variation in the staggered magnetization and $\chi_{\mathrm{t}}$ is the \\ perpendicular susceptibility in the ordered state of the antiferromagnet. The critical behavior of $A$ near $\mathrm{p}_{\mathrm{c}}$ \\ is known. We develop a field-theoretic formalism to calculate $\chi_{t}$. We explicitly calculate $\chi_{t}$ in the mean- \\ field approximation and find that it diverges as $\left|\ln \left(p-p_{c}\right)\right|$, as the concentration $p$ approaches $p_{c}$. Some \\ further scaling arguments yield a scaling relation relating the divergence exponent of $\chi_{t}$ with other known \\ exponents at the percolation critical point. \\ Disciplines \\ Physics
}




\title{
Spin-wave excitations and perpendicular susceptibility of a diluted antiferromagnet near percolation threshold
}

\author{
Deepak Kumar* \\ Department of Physics, University of Wisconsin-Madison, Madison, Wisconsin 53706 \\ A. Brooks Harris \\ Department of Physics, University of Pennsylvania, Philadelphia, Pennsylvania 19104
}

(Received 4 February 1985)

\begin{abstract}
The long-wavelength excitations of a diluted antiferromagnet near the percolation threshold $p_{c}$ are studied. Within the hydrodynamic theory, the excitation frequency depends on two parameters, $A$ and $\chi_{t} . A$ is the stiffness associated with the spatial variation in the staggered magnetization and $\chi_{t}$ is the perpendicular susceptibility in the ordered state of the antiferromagnet. The critical behavior of $A$ near $p_{c}$ is known. We develop a field-theoretic formalism to calculate $\chi_{t}$. We explicitly calculate $\chi_{t}$ in the mean-field approximation and find that it diverges as $\left|\ln \left(p-p_{c}\right)\right|$, as the concentration $p$ approaches $p_{c}$. Some further scaling arguments yield a scaling relation relating the divergence exponent of $\chi_{t}$ with other known exponents at the percolation critical point.
\end{abstract}

\section{INTRODUCTION}

The study of linear dynamical processes on percolation clusters near the percolation threshold is of great current interest. $^{1-3}$ This is a challenging problem, because the infinite cluster over which these processes occur is extremely disordered and ramified. In fact, such clusters are good physical examples of random fractals with nonintegral dimension (within certain length scales). Recently, significant progress has been achieved in the study of a class of processes whose dynamic equations are described by the Laplacian operator or its finite-difference analog. ${ }^{4}$ The physical examples of such processes include ferromagnetic spin waves, phonons, diffusion of a classical particle or exciton, resistor networks, and disordered superconductors. Due to the similarity of the dynamical equations, simple correspondences exist between various physical processes and they are governed by essentially the same exponents and scaling laws.

To give an example, the dispersion relation for the ferromagnetic spin waves at small wave vectors $q\left(q<<\xi_{p}^{-1}\right.$, where $\xi_{p}$ is the percolation correlation length) is given by

$$
\omega(q)=D(p) q^{2},
$$

with $D(p)$ vanishing at the percolation threshold $p_{c}$ like $\left(p-p_{c}\right)^{\mu}$. It has been shown that the spin-wave problem is related to the dilute-resistor-network problem, ${ }^{5}$ and that the index $\mu$ is related to the index $t$, with which the conductivity of the resistor network vanishes at $p_{c}$. The resistor-network problem, in turn, is related to the diffusion problem, or the random-walk problem, which relationship gives rise to the equation

$$
\mu=t-\beta_{p}=v_{p} \theta,
$$

where $\theta$ is the index associated with the diffusion on percolation clusters (infinite cluster) in the following way: ${ }^{6}$

$$
\left\langle r^{2}\right\rangle \propto T^{2 /(2+\theta)},
$$

where $\left\langle r^{2}\right\rangle$ is the mean-square average of the distance a particle diffuses in time $T$. The relationship between these three different physical processes can also be described by saying that all these exponents are governed by the spectral dimension $\overline{\bar{d}}^{7,8}$ of the infinite percolation cluster (IPC), the spectral dimension being defined in terms of density of states as

$$
\rho(\epsilon) \propto \epsilon^{\overline{\bar{d}} / 2-1} .
$$

The relationship between $\overline{\bar{d}}, \theta$, and the fractal dimension $\bar{d}$ of IPC is $\overline{\bar{d}}=2 \bar{d} /(2+\theta)$ and $\bar{d}=d-\beta_{p} / v_{p}$. Through the use of the principle of dynamic scaling, one can further relate the dynamic exponents $z$ of various magnetic models with spectral dimension $\overline{\bar{d}}$ or $\theta .^{9}$

The purpose of the present paper is to study the linearized excitations of a diluted isotropic antiferromagnet in the long-wavelength limit. The antiferromagnetic excitations do not belong to the class mentioned in the above paragraph. The reason for this, as first pointed out by Harris and Kirkpatrick, ${ }^{10}$ can be best understood in terms of the hydrodynamic theory. The energy functional for the long-wavelength antiferromagnetic excitations can be written in terms of the transverse components of sublattice magnetizations $\mathbf{m}_{a}(\mathbf{r})$ and $\mathbf{m}_{b}(\mathbf{r})$ as follows: ${ }^{10}$

$$
\begin{gathered}
E=\sum_{\alpha=x, y} \int d^{d} r\left(\frac{A}{2 M_{s}^{2}}\left|\nabla\left(m_{a}^{\alpha}-m_{b}^{\alpha}\right)\right|^{2}+\frac{H_{E}}{2 M_{s}}\left(m_{a}^{\alpha}+m_{b}^{\alpha}\right)^{2}\right. \\
\left.+\frac{\Gamma}{2 M_{s}}\left[\left(m_{a}^{\alpha}\right)^{2}+\left(m_{b}^{\alpha}\right)^{2}\right]\right)
\end{gathered}
$$

where $M_{s}$ is the sublattice magnetization which is parallel (or antiparallel) to the $z$ axis. $A, H_{E}$, and $\Gamma$ are phenomenological constants which depend upon the concentration of magnetic moments and can also be easily expressed in terms of a microscopic Heisenberg model. The constant $A$ is a measure of the energy needed to create a

(C) 1985 The American Physical Society 
spatial variation in the staggered magnetization, which is the order parameter and is related, like the corresponding quantity in the ferromagnetic case, to the conductance of a related resistor network. Therefore, near $p_{c}$, $A \propto\left(p-p_{c}\right)^{t}$. The quantity $H_{E}$, called the exchange stiffness, measures the resistance to the creation of a uniform magnetization in the perpendicular direction to the sublattice magnetization by an applied field. This quantity has no analog in the problems discussed in the first paragraph. $H_{E}$ is related to the perpendicular susceptibility $\chi_{t}$ by the relation $\chi_{t}=M_{s} / H_{E}$. As the percolation concentration is approached, $H_{E}$ and $\chi_{t}$ show a critical behavior and this affects the excitation frequency, as for the isotropic antiferromagnet (i.e., $\Gamma=0$ ); the excitation frequency is given by

$$
(\omega / \gamma)^{2}=\frac{2 A}{\chi_{t}} q^{2}
$$

where $\gamma$ is the gyromagnetic ratio. It is the critical behavior of $\chi_{t}$, which gives this problem a new character. The true nature of the divergence of $\chi_{t}$ was first elucidated by Harris and Kirkpartrick (HK), ${ }^{10}$ who found numerically that $\chi_{t} \propto\left(p-p_{c}\right)^{-\tau}$ with $\tau=0.5$ in three dimensions. The increase in susceptibility for isotropic antiferromagnets has also been observed experimentally. ${ }^{11,12}$ Breed et al. ${ }^{11}$ extrapolated their results to zero temperature and found that perpendicular susceptibility diverges as $p \rightarrow p_{c}$.

The reason for the divergence of the perpendicular response of the infinite cluster (IC) near $p_{c}$ can be understood in the following physical terms. ${ }^{10}$ We divide the infinite cluster into cells of linear size $L$ which are of order $\xi_{p}$. Considering one such cell, we note that due to fluctuations in site occupation, it is unlikely that the number of occupied $A$ sites equals the number of occupied $B$ sites within the cell. As a result, this cell has a net moment which would turn along the direction of the applied field without any resistance, if the connections of this cell with the rest of the IC were severed. The sublattice magnetizations in the IC are balanced on the whole, so the uncompensated moments of the various cells of the IC are constrained by their neighboring cells and show only a finite response. But as the percolation threshold is approached, the net moment of the typical cell is proportional to $\xi_{p}^{d / 2}$ and the connectivity between the neighboring cells weakens, making it conceivable that $\chi_{t}$ diverges as $p \rightarrow p_{c}$. Denoting the divergence index of $\chi_{t}$ by $\tau$ and using the above considerations, HK (Ref. 10) derived the following heuristic relation for $\tau$ :

$$
\tau \geq t-\beta_{p}-(d-2) \nu_{p},
$$

which gives $\tau \geq 0$ in the mean-field limit. Our mean-field calculations, described in this paper, confirm the equality sign.

In this paper, we shall be interested only in the excitations of very long wavelength, for which the hydrodynamic theory is valid. For such calculations the main relevant quantity to be calculated, as far as critical behavior at the percolation threshold is concerned, is the perpendicular susceptibility. Thus, the main body of this paper describes a calculation of the zero-temperature perpendicular susceptibility $\chi_{t}$ of a uniaxially anisotropic antifer- romagnet. Even though we are interested in the excitations of an isotropic antiferromagnet, introduction of the uniaxial anisotropy serves an important purpose. It enables us to subtract off the paramagnetic response due to finite clusters which have a net moment because of unequal occupation of the two sublattices of the antiferromagnet. If the anisotropy is taken to be zero, this response becomes infinite at zero temperature and hides the relevant quantity of interest. We also show that the same purpose can also be achieved by working at finite but small temperatures.

The perpendicular susceptibility $\chi_{t}$ can be determined by inverting a system of linear equations, which after a simple transformation are analogous to the voltage equations for the corresponding diluted resistor network. In fact, we show that this problem is analogous to determining the resistive response to an applied staggered field. Our treatment is based on a method, developed by Stephen, ${ }^{13}$ to tackle the resistor-network problem. We make explicit calculations of the susceptibility in the mean-field limit and then present some tentative generalizations based on scaling theory.

The formulation made by Stephen converts the problem into a statistical field theory involving an infinite number of order parameters. Hence, the mean-field approximation to the problem is also nontrivial and remains intractable unless some further perturbative expansion is developed. A natural expansion parameter turns out to be $J^{-1}$, where $J$ is the strength of the exchange interaction. This is due to the circumstance (that we can establish on general ground) that the perpendicular susceptibility of the diluted antiferromagnet is proportional to $J^{-1}$, after the paramagnetic contributions due to uncompensated finite clusters have been subtracted off. Thus, we need just the first two terms of the expansion in $J^{-1}$ to obtain an exact result.

The zero-order term corresponding to the limit $J=\infty$ is already very interesting. In this limit, our mean-field equations reduce to the mean-field theory of the pure percolation problem ${ }^{13}$ in the presence of a staggered field. In the case of percolation, the staggered field couples to fluctuations corresponding to the difference in occupation of the two sublattices. These are just the fluctuations that were identified physically as being the cause for the divergence of perpendicular susceptibility by Harris and Kirkpatrick. ${ }^{10}$ This is a particularly appealing feature of the present formalism.

This paper is organized as follows. In Sec. II, we present the basic equations needed to determine $\chi_{t}$. Using these equations, we establish the general form of $\chi_{t}$ in two cases: namely, (a) at zero temperature and finite anisotropy, and (b) at finite, but small temperature, and zero anisotropy. We also exhibit how $\chi_{t}$ is related to the staggered resistance. In Sec. III, we set up the replica formalism for $\chi_{t}$, in close analogy to the work of Stephen. In Sec. IV, we derive the mean-field equations and show how, in the $J=\infty$ limit, they describe the percolation problem in the staggered field. Two appendixes, A and B, supplement this section by establishing the equivalence of this formalism to the mean-field theory of the Potts model in a staggered field. Appendix A describes how the 
staggered field couples to the fluctuations in $\left(n_{A}^{c}-n_{B}^{c}\right)$, where $n_{A}^{c}\left(n_{B}^{c}\right)$ denote the number of $A(B)$ sites in a cluster $c$. Appendix $B$ describes the relevant mean-field theory for the Potts model. In Sec. V, we develop a $J^{-1}$ expansion and calculate the susceptibility to order $J^{-1}$. Some details of this calculation are relegated to Appendix C. Section $\mathrm{V}$ concludes with some tentative scaling arguments which go beyond the mean-field results.

\section{PERPENDICULAR SUSCEPTIBILITY: GENERAL FORMULATION}

We consider a two-sublattice bond-diluted antiferromagnet described by the Hamiltonian

$\mathscr{H}=\sum_{\langle i j\rangle} J_{i j} \mathbf{S}_{i} \cdot \mathbf{S}_{j}-\Gamma \sum_{i}\left(S_{i}^{z}\right)^{2}-\left(g \mu_{B}\right) \sum_{i} H_{i}^{x} S_{i}^{x}$,

where the sum $\langle i j\rangle$ indicates that $i$ and $j$ are to be summed over nearest-neighbor pairs of sites on a lattice whose coordination number is $z$. The lattice is divisible into two sublattices denoted by $A$ and $B$, and the nearest neighbor of sites on the $A$ sublattice lie on the $B$ sublattice, and vice versa. $J_{i j}$ 's are independent random variables, each of which takes values $J$ with the probability $p$ and the value 0 with the probability $1-p$. The second term in the Hamiltonian corresponds to uniaxial anisotropy and is kept here for a calculational purpose. In a randomly diluted system there are clusters in which the number of $A$ sites does not equal the number of $B$ sites. Such clusters, due to their net moment, contribute an infinite response at zero temperature in the absence of the anisotropy term. Furthermore, the anisotropy term stabilizes the Neel ground state in the $z$ direction for all clusters. The last term in (2.1) corresponds to a site-dependent external transverse field.

For small external fields, we can expand about the Néel state by writing

$$
\mathbf{S}_{i}=S\left[\epsilon_{i}\left(1-\theta_{i}^{2} / 2\right) \widehat{\mathbf{z}}+\theta_{i} \widehat{\mathbf{x}}\right],
$$

where $\epsilon_{i}=1$ when $i$ is on the $A$ sublattice, $\epsilon_{i}=-1$ when $i$ is on the $B$ sublattice, and $\theta_{i}$ 's are small deviations caused by the field. The energy in terms of $\theta_{i}$ 's can be written as

$$
\begin{aligned}
E= & -\sum_{i, j} J_{i j} S^{2}+\frac{1}{2} S^{2} \sum_{\langle i j\rangle} J_{i j}\left(\theta_{i}+\theta_{j}\right)^{2}+\frac{\Gamma S^{2}}{2} \sum_{i} \theta_{i}^{2} \\
& -g \mu_{B} S \sum_{i} H_{i}^{x} \theta_{i}
\end{aligned}
$$

The equilibrium deviations obtained by minimizing $E$ are given by

$$
\sum_{j} \widetilde{B}_{i j} \theta_{j}=\left(g \mu_{B} / S\right) H_{i}^{x}
$$

where

$$
\widetilde{B}_{i j}=\left[\sum_{k} J_{i k}+\Gamma\right) \delta_{i j}+J_{i j} .
$$

The perpendicular susceptibility $\chi_{t}$ is now given (setting $\left.H_{i}^{x}=H^{x}\right)$ by

$$
\chi_{t}=\left(g \mu_{B} S\right) \sum_{i} \theta_{i} / H^{x}=\left(g \mu_{B}\right)^{2} \sum_{i, j}\left(\widetilde{B}^{-1}\right)_{i j}
$$

For the diluted system, the sum would break up into sums over distinct physical clusters in the system, and we may write

$$
\hat{\chi}_{t}=\chi_{t} /\left(g \mu_{B}\right)^{2}=\sum_{c} \sum_{i, j \in c}\left(\widetilde{B}^{-1}\right)_{i j},
$$

where the second sum runs over sites within the cluster $c$.

At this point it proves useful to make a change in variables, so that the analogy of the problem to the diluted resistor network becomes evident. For this purpose, we define

$$
\psi_{i}=\epsilon_{i} \theta_{i}
$$

and

$$
\begin{aligned}
B_{i j} & =\epsilon_{i} \epsilon_{j} \widetilde{B}_{i j}=\left[\sum_{k} J_{i k}+\Gamma\right] \delta_{i j}-J_{i j} \\
& =J G_{i j}^{-1}+\Gamma \delta_{i j}
\end{aligned}
$$

The matrix $\underline{G}$, defined in Eq. (2.10), is related to the diluted resistor network defined by treating the occupied bonds of the above system as a unit conductance. If $\left|\phi_{\lambda}\right\rangle$ and $\lambda$ denote, respectively, the eigenvectors and the corresponding eigenvalues of the matrix $\underline{G}^{-1}$, then we may write

$$
\begin{aligned}
\hat{\chi}_{t} & =\sum_{i, j} \epsilon_{i} \epsilon_{j}\left(B^{-1}\right)_{i j} \\
& =\sum_{\lambda} \frac{\left\langle\epsilon \mid \phi_{\lambda}\right\rangle^{2}}{J \lambda+\Gamma},
\end{aligned}
$$

where $|\epsilon\rangle$ is a column matrix whose elements are $\epsilon_{i}$. As the matrix $\underline{G}^{-1}$ has zero as its lowest eigenvalue, we may write

$$
\chi_{t}=\frac{\left\langle\epsilon \mid \phi_{0}\right\rangle^{2}}{\Gamma}+\frac{1}{J} \sum_{\lambda \neq 0} \frac{\left\langle\epsilon \mid \phi_{\lambda}\right\rangle^{2}}{\lambda},
$$

where we have taken $J \gg \Gamma$ and hence neglected $\Gamma$ in the second term. If we set

$$
|\widetilde{\epsilon}\rangle=|\epsilon\rangle-\left\langle\epsilon \mid \phi_{0}\right\rangle\left|\phi_{0}\right\rangle,
$$

we may rewrite Eq. (2.13) as

$$
\hat{\chi}_{t}=\frac{\left\langle\epsilon \mid \phi_{0}\right\rangle^{2}}{\Gamma}+\frac{1}{J}\langle\widetilde{\epsilon}|G| \widetilde{\epsilon}\rangle .
$$

Note that $G$ is defined only in the subspace which is orthogonal to $\left|\phi_{0}\right\rangle$, corresponding to zero eigenvalue. To appreciate the significance of this result let us apply it to a single cluster. Noting that $\left|\phi_{0}\right\rangle=(1,1, \ldots, 1) / \sqrt{n^{c}}$, we find

$$
\hat{\chi}_{t}^{c}=\frac{\left(n_{A}^{c}-n_{B}^{c}\right)^{2}}{n^{c} \Gamma}+\frac{1}{J} \sum_{i, j \in c}\left(\epsilon_{i}-\bar{\epsilon}\right) G_{i j}\left(\epsilon_{j}-\bar{\epsilon}\right),
$$

where $n_{A}^{c}\left(n_{B}^{c}\right)$ are the number of $A(B)$ sites in the cluster, $n^{c}=n_{A}^{c}+n_{B}^{c}$, and

$$
\bar{\epsilon}=\left(n_{A}^{c}-n_{B}^{c}\right) / n^{c} \text {. }
$$

Equation (2.15) shows that a cluster with net moment will have two types of contributions to susceptibility, one of them dependent on its net moment and inversely propor- 
tional to $\Gamma$, and the second one the usual antiferromagnetic response proportional to $J^{-1}$ (for $J \gg \Gamma$ ).

Rather similar considerations can also be made at nonzero temperatures, which are such that $k_{B} T \ll J$. At such temperatures, each cluster will be in the Néel state. If the cluster has a net moment, its paramagnetic response dominates and

$$
\hat{\chi}_{t}^{c}=\frac{\left(n_{A}^{c}-n_{B}^{c}\right)^{2} S^{2}}{3 k_{B} T} .
$$

If the moment of the cluster is balanced, one can calculate the response by making an expansion about the Neel state as follows. We write for each spin,

$$
\mathbf{S}_{i}=\epsilon_{i}\left[x_{i} \widehat{\mathbf{x}}+y_{i} \hat{\mathbf{y}}+\left(1-x_{i}^{2}-y_{i}^{2}\right)^{1 / 2} \widehat{\mathbf{z}}\right],
$$

and express energy to quadratic order in $x_{i}$ 's and $y_{i}$ 's. This yields

$$
\begin{aligned}
E= & E_{0}+S^{2} \sum_{i, j \in c} J_{i j}\left[\left(x_{i}-x_{j}\right)^{2}+\left(y_{i}-y_{j}\right)^{2}\right] \\
& -g \mu_{B} S \sum_{i \in c} H_{i}^{x} \epsilon_{i} x_{i} .
\end{aligned}
$$

From this expression, it follows in a straightforward manner that

$$
\hat{\chi}_{t}^{c}=\frac{1}{J} \sum_{i, j \in c} \epsilon_{i} \epsilon_{j} G_{i j}
$$

Thus, the susceptibility of the total system may be written as

$$
\hat{\chi}_{t}=\sum_{c} \frac{\left(n_{c}^{A}-n_{c}^{B}\right)^{2} S^{2}}{3 k_{B} T}+\frac{1}{J} \sum_{c}^{\prime} \sum_{i, j \in c} \epsilon_{i} \epsilon_{j} G_{i j},
$$

where the second sum is restricted to balanced clusters only.

It may be of interest to point out that the second term in Eqs. (2.15) and (2.21) may also be regarded as the staggered resistance of the corresponding diluted resistance network. If an external current $I$ is introduced at site $n$ and taken out at site $m$, then a resistance $R_{n m}$ may be defined from the following equation:

$$
R_{n m}=\left(V_{n}-V_{m}\right) / I,
$$

where $V_{l}$ denotes voltage at lattice site $l$. It then follows from Kirchoff's laws that

$$
R_{n m}=G_{m m}+G_{n n}-G_{m n}-G_{n m}
$$

and

$$
\sum_{i, j}\left(\epsilon_{i}-\bar{\epsilon}\right)\left(\epsilon_{j}-\bar{\epsilon}\right) G_{i j}=-\sum_{i, j}\left(\epsilon_{i}-\bar{\epsilon}\right) R_{i j}\left(\epsilon_{j}-\bar{\epsilon}\right) / 2 .
$$

Note, further, that finite temperature at zero anisotropy or finite anisotropy at zero temperature serve the same purpose of isolating the paramagnetic response from the uncompensated clusters.

\section{REPLICA FORMALISM FOR PERPENDICULAR SUSCEPTIBILITY}

In this section we set up a replica formalism along the lines of Stephen's treatment ${ }^{13}$ of random resistor networks. For this purpose, we consider the partition function

$$
Z\left\{h_{i}\right\}=\int \prod_{i} d \theta_{i} \exp \left[-\frac{1}{2} \sum_{i, j} \widetilde{B}_{i j} \theta_{i} \theta_{j}+\sum_{i} h_{i} \theta_{i}\right] .
$$

Since

$$
Z=\frac{(2 \pi)^{N / 2}}{(\operatorname{det} B)^{1 / 2}} \exp \left[\frac{1}{2} \sum_{i, j} h_{i} \widetilde{B}_{i j}^{-1} h_{j}\right],
$$

it follows that

$$
\chi_{t}=\left.\frac{\partial^{2}}{\partial h^{2}}\left\langle\left(\ln Z\left\{h_{0}=h\right\}\right)\right\rangle_{c}\right|_{h=0},
$$

where \langle\rangle$_{c}$ denotes configuration averaging. Thus, to calculate the configuration-averaged susceptibility, we need to calculate $\langle\ln Z\rangle_{c}$, for which purpose the wellknown replica trick can be employed. We write

$$
\langle\ln Z\rangle_{c}=\left.\frac{\partial}{\partial n}\left\langle Z^{n}\right\rangle_{c}\right|_{n=0}
$$

and

$$
Z^{n}=\int \prod_{i, \alpha} d \theta_{i}^{\alpha} \exp \left[-\frac{1}{2} \sum_{i, j} \sum_{\alpha=1}^{n} \widetilde{B}_{i j} \theta_{i}^{\alpha} \theta_{j}^{\alpha}+\sum_{i} h_{i}^{\alpha} \theta_{i}^{\alpha}\right] .
$$

On performing the configuration averaging at this stage, one obtains

$$
\left\langle Z^{n}\right\rangle_{c}=\int \prod_{i, \alpha} d \theta_{i}^{\alpha} \exp \left(\sum_{i} \mathbf{h}_{i} \cdot \boldsymbol{\theta}_{i}-\frac{\Gamma}{2} \sum_{i} \theta_{i}^{2}\right] \prod_{\langle i j\rangle}\left[1-p+p \exp \left[-\frac{J}{2}\left(\theta_{i}+\boldsymbol{\theta}_{j}\right)^{2}\right]\right),
$$

where $\theta_{i}$ stands for replica variables $\theta_{i}^{\alpha}, \alpha=1, n$. We now introduce the variables $\psi_{i}$ from Eq. (2.8) to write

$$
\left\langle Z^{n}\right\rangle_{c}=(1-p)^{N z / 2} \int \prod_{i} d \psi_{i} \exp \left[\sum_{i} \epsilon_{i} \mathbf{h}_{i} \cdot \psi_{i}-\frac{\Gamma}{2} \sum_{i} \psi_{i}^{2}+\sum_{\langle i j\rangle} \ln \left(1+v e^{-(J / 2)\left(\boldsymbol{\psi}_{i}-\boldsymbol{\psi}_{j}\right)^{2}}\right)\right],
$$

where $v=(p /(1-p)$. The replicated partition function in Eq. (3.7) is analogous to a similar quantity for the diluted resistor network. ${ }^{13}$ Following Stephen, we write

$$
\ln \left(1+v e^{-(J / 2)\left(\boldsymbol{\psi}_{i}-\boldsymbol{\psi}_{j}\right)^{2}}\right)=\frac{1}{z} \sum_{\mathbf{p}} B_{\mathbf{p}} e^{i \mathbf{p} \cdot\left(\boldsymbol{\psi}_{i}-\boldsymbol{\psi}_{j}\right)}
$$


where $\sum_{p}$ denotes $\int d \mathbf{p} /(2 \pi)^{n / 2}$ and

$$
B_{\mathrm{p}}=z \sum_{l=1}^{\infty}(-1)^{l+1} \frac{v^{l}}{l} \frac{1}{(l J)^{n / 2}} \exp \left(-\frac{p^{2}}{2 J l}\right) .
$$

Substituting Eq. (3.9) into (3.8), we get

$$
\left\langle Z^{n}\right\rangle_{c}=\int d \psi_{i} \exp \left(\sum_{i} \epsilon_{i} \mathbf{h}_{i} \cdot \psi_{i}-\frac{\Gamma}{2} \sum_{i} \psi_{i}^{2}+\frac{1}{2} \sum_{\mathrm{p}} B_{\mathrm{p}} \sum_{i j} A_{i j} Q_{\mathrm{p}}\left(\psi_{i}\right) Q_{-\mathrm{p}}\left(\psi_{j}\right)\right),
$$

where $Q_{\mathrm{p}}\left(\boldsymbol{\psi}_{i}\right)=\exp \left(i \mathbf{p} \cdot \boldsymbol{\psi}_{i}\right)$ and

$$
A_{i j}=\left\{\begin{array}{l}
1 / z \text { if } i \text { and } j \text { are nearest neighbors } \\
0 \text { otherwise. }
\end{array}\right.
$$

Performing a Gaussian transformation on Eq. (3.10) enables us to write

$$
\begin{aligned}
\left\langle Z^{n}\right\rangle & =\int \prod_{p, i} d s_{i}(\mathbf{p}) \int \prod_{i} d \psi_{i} \exp \left(-\frac{1}{2} \sum_{\mathbf{p}} B_{\mathbf{p}} \sum_{i, j} A_{i j}^{-1} s_{i}(\mathbf{p}) s_{j}(-\mathbf{p})+\sum_{i, \mathbf{p}} B_{p} s_{i}(\mathbf{p}) Q_{-\mathbf{p}}\left(\boldsymbol{\psi}_{j}\right)+\sum_{i} \epsilon_{i} \mathbf{h}_{i} \cdot \boldsymbol{\psi}_{i}-\frac{\Gamma}{2} \sum_{i} \psi_{i}^{2}\right) \\
& =\int \prod_{\mathbf{p}, i} d s_{i}(\mathbf{p}) \exp \left(-\frac{1}{2} \sum_{\mathbf{p}} B_{\mathbf{p}} \sum_{i j} A_{i j}^{-1} s_{i}(\mathbf{p}) s_{j}(-\mathbf{p})+\sum_{i} u\left[s_{i}(\mathbf{p})\right]\right) \\
& =\int \prod_{i, \mathbf{p}} d s_{i}(\mathbf{p}) \exp \left\{\mathscr{H}_{\mathrm{eff}}\left[s_{i}(\mathbf{p})\right]\right\},
\end{aligned}
$$

where

$$
\exp \left\{u\left[s_{i}(\mathbf{p})\right]\right\}=\int d \boldsymbol{\psi} \exp \left[\epsilon_{i} \mathbf{h}_{i} \cdot \boldsymbol{\psi}-\frac{\Gamma \psi^{2}}{2}+\sum_{\mathbf{p}} B_{\mathbf{p}} s_{i}(\mathbf{p}) e^{-i \mathbf{p} \cdot \psi}\right] .
$$

Equation (3.13) is a very convenient form to apply the machinery of field-theoretic and usual statistical methods to this problem. ${ }^{14}$ The field-theoretic variables $s_{i}(\mathbf{p})$ are closely related to our original $\psi_{i}$ variables through the relations

$$
\left\langle s_{i}(\mathbf{p})\right\rangle=\sum_{j} A_{i j}\left\langle e^{-i \mathbf{p} \cdot \psi_{i}}\right\rangle
$$

and

$$
\sum_{j} A_{i j}\left\langle\psi_{j}^{\alpha}\right\rangle=\left.(i) \frac{\partial}{\partial p_{\alpha}}\left\langle s_{i}(\mathbf{p})\right\rangle\right|_{\mathbf{p}=0} .
$$

These two relations prove to be very convenient in calculating susceptibility and other physical quantities in this formalism. It turns out that in the mean-field approximation, the more convenient variables to deal with are the Fourier-transformed variables $S_{i}(\mathbf{W})$, defined by

$$
S_{i}(\mathbf{W})=\sum_{\mathbf{p}} e^{i \mathbf{p} \cdot \mathbf{w}} s_{i}(\mathbf{p}) .
$$

Then Eq. (3.16) takes the form

$$
\sum_{j} A_{i j}\left\langle\psi_{j}^{\alpha}\right\rangle=\int \frac{d^{n} W}{(2 \pi)^{n / 2}} W^{\alpha}\left\langle S_{i}(\mathbf{W})\right\rangle .
$$

Let us now consider the situation in a uniform field. A uniform $\mathbf{h}_{i}=\mathbf{h}_{\pi}$ acts like a staggered field for the $\boldsymbol{\psi}_{i}$ or $s_{i}(\mathbf{p})$ variables, and we expect the averages of these variables to assume uniform values on each sublattice, i.e.,

$$
\left\langle\psi_{i}^{\alpha}\right\rangle=\psi_{A(B)}^{\alpha}, \quad i \in A(i \in B)
$$

and

$$
\psi_{A}^{\alpha} \neq \psi_{B}^{\alpha} \text { for } \mathbf{h}_{\pi} \neq 0 .
$$

Equation (3.18) may now be written as

$$
\psi_{A(B)}^{\alpha}=\int \frac{d^{n} W}{(2 \pi)^{n / 2}} W^{\alpha}\left\langle S_{B(A)}(\mathbf{W})\right\rangle .
$$

The perpendicular susceptibility is then given by

$$
\begin{aligned}
\chi_{t} /\left(g \mu_{B}\right)^{2}= & \left.\frac{\partial}{\partial h_{\pi}} \sum_{i} \epsilon_{i}\left\langle\psi_{i}^{\alpha}\right\rangle\right|_{n \rightarrow 0} \\
= & \frac{N}{2} \frac{\partial}{\partial h_{\pi}}\left(\psi_{A}^{\alpha}-\psi_{B}^{\alpha}\right) \\
= & \frac{N}{2} \int \frac{d^{n} W}{(2 \pi)^{n / 2}} \\
& \quad \times\left. W^{\alpha} \frac{\partial}{\partial h_{\pi}}\left\langle S_{B}(\mathbf{W})-S_{A}(\mathbf{W})\right\rangle\right|_{n \rightarrow 0} .
\end{aligned}
$$

We shall employ Eq. (3.23) to calculate $\chi_{t}$ in Sec. V.

\section{MEAN-FIELD THEORY AND THE $J=\infty$ LIMIT}

In this section we calculate $\left\langle Z^{n}\right\rangle_{c}$ in a mean-field approximation ${ }^{13}$ (MFA) and derive self-consistent equations for the order parameters in this approximation. Then we solve these equations in the $J=\infty$ limit and relate the solutions to the percolation problem. The MFA consists of calculating the functional integral in $\left\langle Z^{n}\right\rangle$ by the steepest-descent method, i.e., 


$$
\begin{aligned}
\left\langle Z^{n}\right\rangle_{c} & =\exp \left\{-\mathscr{H}_{\mathrm{eff}}\left[\bar{s}_{i}(\mathbf{p})\right]\right\} \int \prod_{i, p} d s_{i}(\mathbf{p}) \exp \left[-\frac{1}{2} \sum_{i \mathbf{p}, j \mathbf{p}^{\prime}} Q_{i \mathbf{p}, j \mathbf{p}^{\prime}}\left[s_{i}(\mathbf{p})-\bar{s}_{i}(\mathbf{p})\right]\left[s_{j}\left(\mathbf{p}^{\prime}\right)-\bar{s}_{j}\left(\mathbf{p}^{\prime}\right)\right]\right) \\
& =\exp \left\{-\mathscr{H}_{\mathrm{eff}}\left[\bar{s}_{i}(\bar{p})\right]\right\}(\operatorname{det} Q)^{-1 / 2},
\end{aligned}
$$

where

$$
Q_{i \mathbf{p}, j \mathbf{p}^{\prime}}=\frac{\partial^{2} \mathscr{H}_{\mathrm{eff}}}{\partial s_{i}(\mathbf{p}) \partial s_{j}\left(\mathbf{p}^{\prime}\right)} .
$$

The quantities $\bar{s}_{i}(\mathbf{p})$ obtained by maximizing the functional in the exponent are given by

$$
\sum_{j} A_{i j}^{-1} \bar{s}_{j}(-\mathbf{p})=\frac{1}{Z_{1 i}} \int d \psi \exp \left(-i \mathbf{p} \cdot \psi+\epsilon_{i} \mathbf{h}_{i} \cdot \psi-\frac{\Gamma}{2} \psi^{2}+\sum_{p} B_{\mathbf{p}} \bar{s}_{i}(\mathbf{p}) e^{-i \mathbf{p} \cdot \psi}\right),
$$

where

$$
Z_{1 i}=\int d \psi \exp \left[\epsilon_{i} \mathbf{h}_{i} \cdot \psi-\frac{\Gamma}{2} \psi^{2}+\sum_{\mathbf{p}} B_{\mathrm{p}} \bar{s}_{i}(\mathbf{p}) e^{-i \mathbf{p} \cdot \psi}\right]
$$

The best way to solve the mean-field equation (4.3) is to take a Fourier transform with respect to the variable p. This yields

$$
S_{i}(\mathbf{W})=\sum_{j} A_{i j} \frac{1}{Z_{1 j}} \exp \left[\epsilon_{j} \mathbf{h}_{j} \cdot \mathbf{W}-\frac{\Gamma \mathbf{W}^{2}}{2}+\sum_{\mathbf{p}} B_{\mathbf{p}} \cdot \bar{s}_{i}(\mathbf{p}) e^{-i \mathbf{p} \cdot \mathbf{W}}\right] .
$$

$Z_{1 i}$ is a normalizing factor in the sense that

$$
\int \sum A_{i j}^{-1} s_{j}(W) d^{n} W=1 .
$$

Equation (4.5) is a key equation which we employ repeatedly in the rest of the work. We first look for a limit in which the percolation problem is recovered. ${ }^{13}$ Note that when $J=\infty, B_{\mathrm{p}}=z \ln (1+v)=x$ for all $\mathbf{p}$ and Eq. (4.5) becomes

$$
S_{i}(\mathbf{W})=\sum_{j} A_{i j} \frac{1}{Z_{i j}} \exp \left[\epsilon_{j} \mathbf{h}_{j} \cdot \mathbf{W}-\frac{\Gamma \mathbf{W}^{2}}{2}+x S_{j}(\mathbf{W})\right] .
$$

Now we solve Eq. (4.6) in two simple field configurations, (a) $\mathbf{h}_{i}=\epsilon_{i} \mathbf{h}$ and (b) $\mathbf{h}_{i}=\mathbf{h}_{\pi}$. For case (a), Eq. (4.6) becomes site independent and $Z_{1 j}=\exp (x)$, so that

$$
S(\mathbf{W})=\exp \left[\mathbf{h} \cdot \mathbf{W}-\frac{\Gamma \mathbf{W}^{2}}{2}+x(S(\mathbf{W})-1)\right] .
$$

Stephen has solved this equation, ${ }^{15}$ the solution being

$$
S(\mathbf{W})=\sum_{m=1}^{\infty} P_{m} e^{m\left(\mathbf{h} \cdot \mathbf{W}-\Gamma \mathbf{W}^{2} / 2\right)}
$$

with

$$
P_{m}=\frac{(m x)^{m-1}}{m !} \exp (-m x) .
$$

Here $P_{m}$ can be interpreted as the probability that a given site belongs to an $m$-site cluster. Clearly $S(0)$ is the probability that a given site belongs to finite clusters and $P_{\infty}=1-S(0)$ is the usual percolation order parameter, which is such that

$$
P_{\infty}=\left\{\begin{array}{l}
0 \text { for } x<1 \text { or } p<p_{c}, \\
2\left|r_{0}\right| \text { for } x>1 \text { or } p>p_{c},
\end{array}\right.
$$

where $r_{0}=1-x$ and $x=1$ corresponds to $p=p_{c}=1-\exp (-1 / z)$.

Now let us consider case (b). Equation (4.6) for $A$ sites and $B$ sites is different now and may be written as

$$
\begin{aligned}
& S_{A}(\mathbf{W})=\frac{1}{Z_{1 b}} \exp \left[-\mathbf{h}_{\pi} \cdot \mathbf{W}-\frac{\Gamma \mathbf{W}^{2}}{2}+x S_{B}(\mathbf{W})\right], \\
& S_{B}(\mathbf{W})=\frac{1}{Z_{1 a}} \exp \left[\mathbf{h}_{\pi} \cdot \mathbf{W}-\frac{\Gamma \mathbf{W}^{2}}{2}+x S_{A}(\mathbf{W})\right] .
\end{aligned}
$$

We again assert that $z_{1 a}=z_{1 b}=\exp (x)$. This can be seen by establishing the correspondence of these equations with the percolation problem in a staggered field. In Appendix $B$, we describe the mean-field theory of an $s$-state Potts model in a staggered field. This calculation clearly shows that the percolation order parameters $\psi_{A}$ and $\psi_{B}$ obey the same equations as the quantities $P_{A}=1-S_{A}(\mathrm{~W})$ and $P_{B}=1-S_{B}(\mathrm{~W})$, with our choice of $Z_{1 a}$ and $Z_{1 b}$ and taking $\Gamma=0$. To develop further physical significance of the order parameters $\psi_{A}$ and $\psi_{B}$ (or $P_{A}$ and $P_{B}$ ), we consider the diagrammatic expansion for the free energy of a Potts model in a staggered field. The Potts Hamiltonian is ${ }^{14}$

$$
\beta \mathscr{H}=\sum_{i, j} K\left(s \delta_{\sigma_{i} \sigma_{j}}-1\right)-H \sum_{i} \epsilon_{i}\left(s \delta_{\sigma_{i}, 1}-1\right),
$$

where the variables $\sigma_{i}$ at each site assume $s$-distinct values. The partition function can be written as

$$
\begin{aligned}
Z= & s^{-N} e^{-N K z / 2} \operatorname{Tr}_{\left\{\sigma_{i}\right\}} \exp \left[s H \sum_{i} \epsilon_{i}\left(\delta_{\sigma_{i}}, 1-1\right)\right] \\
& \times \prod_{i, j}\left(1+v_{1} \delta_{\sigma_{i}, \sigma_{j}}\right),
\end{aligned}
$$


where $v_{1}=\left(e^{s K}-1\right)$ and is identified to $p /(1-p)$ in the correspondence to the percolation problem in the $s \rightarrow 1$ limit. ${ }^{14}$ Developing a straightforward cluster expansion and taking the $s \rightarrow 1$ limit, one finds that the free energy per site can be written as (see Appendix A for details)

$$
f_{1}=\frac{z K}{2}-1+\sum_{n_{A}, n_{B}} \bar{W}\left(n_{A}, n_{B}\right) e^{-H\left(n_{A}-n_{B}\right)},
$$

where $\bar{W}\left(n_{A}, n_{B}\right)$ denotes the average number of clusters per site containing $n_{A} A$-lattice sites and $n_{B} B$-lattice sites. Thus, $f_{1}$ behaves like a generator for calculating cluster averages $\left(n_{A}-n_{B}\right)$. Clearly,

$$
\begin{aligned}
& \left\langle n_{A}-n_{B}\right\rangle=\sum_{n_{A}, n_{B}}\left(n_{A}-n_{B}\right) \bar{W}\left(n_{A}, n_{B}\right)=-\left.\frac{\partial f_{1}}{\partial H}\right|_{H=0}, \\
& \left\langle\left(n_{A}-n_{B}\right)^{2}\right\rangle=\sum_{n_{A}, n_{B}}\left(n_{A}-n_{B}\right)^{2} \bar{W}\left(n_{A}, n_{B}\right)=\left.\frac{\partial^{2} f_{1}}{\partial H^{2}}\right|_{H=0} .
\end{aligned}
$$

We can now define

$$
\begin{aligned}
& 2 \psi_{A}=1-\sum_{n_{A}, n_{B}} n_{A} \bar{W}\left(n_{A}, n_{B}\right) e^{-H\left(n_{A}-n_{B}\right)}, \\
& 2 \psi_{B}=1-\sum_{n_{A}, n_{B}} n_{B} \bar{W}\left(n_{A}, n_{B}\right) e^{-H\left(n_{A}-n_{B}\right)},
\end{aligned}
$$

so that

$$
\frac{\partial f_{1}}{\partial H}=\left(\psi_{A}-\psi_{B}\right) / 2
$$

This then identifies $\psi_{A}$ and $\psi_{B}$ with the mean-field order parameters introduced in Appendix B.

Due to symmetry between the $A$ and $B$ sublattices, $\left\langle n_{A}-n_{B}\right\rangle=0$ when $H=0$. But $\left\langle\left(n_{A}-n_{B}\right)^{2}\right\rangle$, which describes the fluctuations that are essentially responsible for the divergence of the real susceptibility, should show some critical behavior. We calculate this quantity in the meanfield approximation, using the free-energy expression derived in Appendix B:

$$
\begin{aligned}
& f_{1}= \frac{z K}{2}\left(\psi_{A}+\psi_{B}-\psi_{A} \psi_{B}\right) \\
&+\frac{1}{2}\left(e^{-\left(z K \psi_{B}+H\right)}+e^{-\left(z K \psi_{A}-H\right)}\right), \\
&\left.\frac{\partial^{2} f_{1}}{\partial H^{2}}\right|_{H=0}=\frac{1-\psi}{1+z K(1-\psi)}=\left\langle\left(n_{A}-n_{B}\right)^{2}\right\rangle,
\end{aligned}
$$

where $\psi=\psi_{A}=\psi_{B}$ as $H \rightarrow 0$. This gives

$$
\left\langle\left(n_{A}-n_{B}\right)^{2}\right\rangle=\left\{\begin{array}{l}
\frac{1}{2}+\frac{1}{4} r_{0}, \quad p<p_{c} \\
\frac{1}{2}-\frac{3}{4}\left|r_{0}\right|, \quad p>p_{c}
\end{array}\right.
$$

where $r_{0}=z K-1=x-1$.

\section{CALCULATION OF PERPENDICULAR SUSCEPTIBILITY}

In this section, we return to the mean-field equation (4.5) and use it to calculate $\chi_{t}$. We first consider the $J=\infty$ limit. The consideration of this limit is important for the following reason. For a system with zero moment, like a pure antiferromagnet, $\chi_{t}=0$ in the $J=\infty$ limit. But in a diluted system, due to clusters with net moments, there is a response proportional to $\Gamma^{-1}$. We would like to isolate this part of the response. The mean-field equations in the presence of a uniform field in the $J=\infty$ limit are given by Eqs. (4.11) and (4.12). We recast these in terms of new variables defined as

$$
S_{0}(\mathbf{W})=\frac{1}{2}\left[S_{A}(\mathbf{W})+S_{B}(\mathbf{W})\right]
$$

and

$$
S_{\pi}(\mathbf{W})=\frac{1}{2}\left[S_{B}(\mathbf{W})-S_{A}(\mathbf{W})\right] .
$$

Then Eqs. (4.10) and (4.11) can be written as

$$
\begin{aligned}
& S_{\pi}(\mathbf{W})=S_{0}(\mathbf{W}) \tanh \left[\mathbf{h}_{\pi} \cdot \mathbf{W}-x S_{\pi}(\mathbf{W})\right], \\
& S_{0}^{2}(\mathbf{W})-S_{\pi}^{2}(\mathbf{W})=\exp \left\{-\Gamma \mathbf{W}^{2}+2 x\left[S_{0}(\mathbf{W})-1\right]\right\},
\end{aligned}
$$

when $\mathbf{h}_{\pi}=0, S_{\pi}(\mathbf{W}) \rightarrow 0$ and Eq. (5.4) is similar to Eq. (4.7) with the solution

$$
S_{0}(\mathbf{W})=\sum_{m} P_{m} e^{-m \Gamma \mathbf{W}^{2} / 2} .
$$

We can use Eq. (5.3) to calculate the field derivative of $S_{\pi}(\mathbf{W})$ in the limit $\mathbf{h}_{\pi} \rightarrow 0$,

$$
\left.\frac{\partial S_{\pi}(\mathbf{W})}{\partial h_{\pi}^{\alpha}(\mathbf{W})}\right|_{\mathbf{h}_{\pi} \rightarrow 0}=\chi_{\pi}(\mathbf{W})=\frac{W_{\alpha} S_{0}(\mathbf{W})}{1+x S_{0}(\mathbf{W})} .
$$

Using Eq. (3.23), we find

$$
\hat{\boldsymbol{\chi}}_{t} / N=\left.\int \frac{d^{n} W}{(2 \pi)^{n / 2}} \frac{W_{\alpha}^{2} S_{0}(\mathbf{W})}{1+x S_{0}(\mathbf{W})}\right|_{n \rightarrow 0} .
$$

The $n \rightarrow 0$ limit can be taken by noting that $S_{0}(\mathrm{~W})$ is a function of the magnitude of $\mathbf{W}$. In spherical coordinates,

$$
\begin{aligned}
\hat{\chi}_{t} / N & =\left.\int_{0}^{\infty} d W \frac{W^{n+1} S_{0}(W)}{1+x S_{0}(W)} \cdot \int d \Omega_{n} \cos ^{2} \theta\right|_{n \rightarrow 0} \\
& =\int_{0}^{\infty} d W \frac{W S_{0}(W)}{1+x S_{0}(W)},
\end{aligned}
$$

where $d \Omega_{n}$ denotes the angular integration, which yields unity in the $n \rightarrow 0$ limit. Changing the integration variable to $u=\Gamma W^{2} / 2$ finally gives

$$
\chi_{t} / N\left(g \mu_{B}\right)^{2}=\frac{1}{\Gamma} \int_{0}^{\infty} d u \frac{S_{1}(u)}{1+x S_{1}(u)},
$$

where

$$
S_{1}(u)=\sum_{s=1}^{\infty} P_{s} e^{-s u} .
$$

Noting the fact that $S_{1}(0)^{*} \sim 0(1)$ and $S_{1}(u)$ tends to zero monotonically as $u \rightarrow \infty$, we can argue that the denomina- 
tor in the integral is a slowly varying function lying between $1+x$ and 1 and hence can be replaced by a constant. Thus,

$$
\begin{aligned}
\chi_{t} / N\left(g \mu_{B}\right)^{2} & \geq \frac{1}{\Gamma} \frac{1}{1+x} \sum_{s=1}^{\infty} P_{s} \int_{0}^{\infty} d u e^{-s u} \\
& \geq \frac{1}{\Gamma} \frac{1}{1+x} \sum_{s=1}^{\infty} \frac{1}{s} P_{s} \\
& \geq \frac{1}{2 \Gamma}\left(A_{0}+B_{0}\left|r_{0}\right|^{2-\alpha_{p}}+\cdots\right),
\end{aligned}
$$

where $\alpha_{p}=-1$ in the mean-field approximation. Equation $(5.12)$ follows by noting that $(1 / s) P_{s}$ is the average number of $s$-site clusters. ${ }^{16}$ Equation (5.9) shows that in the $J \rightarrow \infty$ limit, we get a contribution proportional to $\Gamma^{-1}$, which must come from uncompensated clusters.

Next we consider an expansion of the mean-field equations in powers of $J^{-1}$ and calculate the first-order contribution. To the first order in $J^{-1}, B_{\mathrm{p}}=x-p^{2} / b$, where

$$
\frac{1}{b}=\frac{z}{2 J} \sum_{l=1}^{\infty}(-1)^{l+1} \frac{v^{l}}{l^{2}} \text {. }
$$

Substituting (5.12) into Eq. (4.5) and considering the uniform-field case, the mean-field equations for the two sublattices assume the form

$$
\begin{aligned}
& S_{B}(\mathbf{W})=\exp \left[\mathbf{h}_{\pi} \cdot \mathbf{W}-\frac{\Gamma \mathbf{W}^{2}}{2}+x\left[S_{A}(\mathbf{W})-1\right]\right. \\
& \left.+\frac{1}{b} \nabla_{W}^{2} S_{A}(\mathbf{W})\right] \\
& S_{A}(\mathbf{W})=\exp \left[-\mathbf{h}_{\pi} \cdot \mathbf{W}-\frac{\Gamma \mathbf{W}^{2}}{2}+x\left[S_{B}(\mathbf{W})-1\right]\right. \\
& \left.+\frac{1}{b} \nabla_{W}^{2} S_{B}(\mathbf{W})\right] \text {. }
\end{aligned}
$$

Repeating steps between Eqs. (5.2) and (5.6), we find that $\chi_{\pi}(\mathbf{W})$ defined in Eq. (5.6) and $S_{0}(\mathbf{W})$ in the $h_{\pi} \rightarrow 0$ limit now obey the equations

$$
\frac{1}{b} \nabla_{W}^{2} \chi_{\pi}(\mathbf{W})+\left(x+\frac{1}{S_{0}(\mathbf{W})}\right) \chi_{\pi}(\mathbf{W})=W_{\alpha}
$$

and

$$
S_{0}(\mathbf{W})=\exp \left[-\frac{\Gamma \mathbf{W}^{2}}{2}+x\left[S_{0}(\mathbf{W})-1\right]+\frac{1}{b} \nabla_{W}^{2} S_{0}(\mathbf{W})\right] .
$$

Both $\chi_{\pi}$ and $S_{0}$ can be expanded in powers of $b^{-1}$, i.e.,

$$
\begin{aligned}
& \chi_{\pi}(\mathbf{W})=\chi_{\pi}^{(1)}+b^{-1} \chi_{\pi}^{(2)}+\cdots, \\
& S_{0}(\mathbf{W})=S_{0}^{(1)}+b^{-1} S_{0}^{(2)}+\cdots .
\end{aligned}
$$

Substituting these expansions into Eqs.(5.15) and (5.16) one finds naturally enough that $S_{0}^{(1)}(\mathbf{W})$ and $\chi_{\pi}^{(1)}(\mathbf{W})$ are given by Eqs. (5.5) and (5.6), respectively, and

$$
\begin{aligned}
& S_{0}^{(2)}(\mathbf{W})= \frac{S_{0}^{(1)}(\mathbf{W})}{1-x S_{0}^{(1)}(\mathbf{W})} \nabla_{W}^{2} S_{0}^{(1)}(\mathbf{W}) \\
& \chi_{\pi}^{(2)}(\mathbf{W})=\frac{S_{0}^{(1)}(\mathbf{W})}{1+x S_{0}^{(1)}(\mathbf{W})}\left[\frac{S_{0}^{(2)}(\mathbf{W})}{\left[S_{0}^{(1)}(\mathbf{W})\right]^{2}} \chi_{\pi}^{(1)}(\mathbf{w})\right. \\
&\left.-\nabla_{W}^{2} \chi_{\pi}^{(1)}(\mathbf{W})\right] .
\end{aligned}
$$

Furthermore,

$$
\begin{aligned}
\hat{\chi}_{t} / N & =\int d^{n} W W^{\alpha} \chi_{\pi}(\mathbf{W}) \\
& =\chi_{t}^{(0)}+\frac{1}{b}\left(\chi_{t}^{(1)}+\chi_{t}^{(2)}\right),
\end{aligned}
$$

where $\chi_{t}^{(0)}$ is given in Eq. (5.11), and

$$
\chi_{t}^{(1)}=-\int d^{n} W \frac{W^{\alpha} S_{0}^{(1)}(\mathbf{W})}{1+x S_{0}^{(1)}(\mathbf{W})} \nabla_{W}^{2}\left[\frac{W_{\alpha} S_{0}^{(1)}(\mathbf{W})}{1+x S_{0}^{(1)}(\mathbf{W})}\right]
$$

and

$$
\chi_{t}^{(2)}=\int d^{n} W \frac{W_{\alpha}^{2} S_{0}^{(1)} \mathbf{W} \nabla_{W}^{2}\left[S_{0}^{(1)}(\mathbf{W})\right]}{\left[1+x S_{0}^{(1)}(\mathbf{W})\right]^{2}\left[1-x S_{0}^{(1)}(\mathbf{W})\right]} .
$$

To evaluate these integrals, we proceed as before, by first going into spherical coordinates and then taking the $n \rightarrow 0$ limit. Changing the integration variable to $u=\Gamma \mathbf{W}^{2} / 2$ and replacing $1+x S_{1}(\mathbf{W})$ by a constant $C_{1}$, we find

$$
\chi_{t}^{(1)}=C_{1}^{-2}\left[\left(\sum_{s} P_{s}\right]^{2}+2 \int_{0}^{\infty} d u S_{1}^{\prime}(u)\left[S_{1}^{\prime}(u)-S_{1}(u)\right]\right]
$$

$\chi_{t}^{(2)}=2 C_{1}^{-2} \int_{0}^{\infty} d u \frac{u S_{1}(u) S_{1}^{\prime \prime}(u)}{1-x S_{1}(u)}$

The first term of $\chi_{t}^{(1)}$ is clearly finite. The integrals yield divergent contributions as $\left|r_{0}\right| \rightarrow 0$, due to the fact that $S_{1}^{\prime}(0) \propto\left|r_{0}\right|^{-1}, S_{1}^{\prime \prime}(0) \propto\left|r_{0}\right|^{-3}$, and $1-x S_{1}(0) \propto\left|r_{0}\right|$. The singular behavior of the integrals can be elicited if we note that $S_{1}(u)$ and its derivatives fall off exponentially as $u \rightarrow \infty$.So the significant contribution to the integrals come from small $u$ values. Near the percolation threshold, for small $u$ values, we can employ the scaling form of $S_{1}(u)$, given by ${ }^{13}$

$$
S_{1}(u)=1+r_{0} \mp\left(r_{0}^{2}+2 u\right)^{1 / 2}, \quad r_{0} \gtrless 0 .
$$

Since the scaling form is not valid at large $u$, the integral should be cutoff at a value of $O(1)$, which we choose to be $\frac{1}{2}+r_{0}$, a value at which $S_{1}(u)$ becomes zero. With the use of Eq. (5.26), the integrations in Eqs. (5.24) and (5.25) can be done analytically to yield

$$
\begin{array}{r}
\chi_{t}^{(1)}+\chi_{t}^{(2)}=C_{1}^{-2}\left\{\left[\sum_{s} P_{s}\right]^{2}+\left[-\ln \left|r_{0}\right|+\ln \left(1+r_{0}\right)-\left(\frac{3}{2}+2 r_{0}\right)\right]+\right. \\
-\ln \left|r_{0}\right|+\left[\frac{1}{r_{0}}-\frac{\left(1-r_{0}\right)}{r_{0}^{2}} \ln \left(1+r_{0}\right)\right] \\
\left.\left.+\frac{2-r_{0}+2 r_{0}^{2}}{2\left(1-r_{0}\right)}-\frac{2 r_{0}}{\left(1-r_{0}\right)\left(1+2 r_{0}\right)}\right]\right\} .
\end{array}
$$


Thus, for small $\left|r_{0}\right|$, the leading singularity of $\chi_{t}$ is $\ln \left(1 /\left|r_{0}\right|\right)$. We can now write down the dominant contributions,

$$
\begin{aligned}
\hat{\chi}_{t} / N= & \frac{1}{\Gamma}\left(A_{0}+B_{0}\left|r_{0}\right|^{3}+\cdots\right) \\
& +\frac{1}{J}\left[A_{1}+B_{1} \ln \left(1 /\left|r_{0}\right|\right)+\cdots\right] .
\end{aligned}
$$

For the calculation of spin-wave dispersion, we need the response of the infinite cluster only. One expects the singular part of the response, which dominates near percolation threshold, to come from the infinite cluster. Since it is independent of $\Gamma$, it is clearly relevant for the excitations of the isotropic antiferromagnet. The logarithmic divergence that we obtain corresponds to $\tau=0$, which is in accord with the value obtained from the relation (1.7) when $d$ is set to 6 ; the upper critical dimensionality and the other exponents are given their mean-field values.

To conclude this paper, we present some tentative scaling arguments which enable us to go beyond the meanfield calculations. Our results are based on the exact equations (2.15) and (2.21). To develop our arguments, one of us has obtained ${ }^{17}$ a scaling form for the average cluster numbers $W\left(n_{A}^{c}, n_{B}^{c}, p\right)$ defined in Eq. (4.15). This is a generalization of the scaling formula $W\left(n=n_{A}^{c}+n_{B}^{c}, p\right){ }^{16}$ Recall

$$
\begin{aligned}
W(n, p) & \approx n^{-\tau_{1}} f_{1}\left[n\left(p-p_{c}\right)^{\beta+\gamma}\right] \\
& \approx\left(p-p_{c}\right)^{\gamma+2 \beta} \frac{1}{n} f_{2}\left[n\left(p-p_{c}\right)^{\beta+\gamma}\right],
\end{aligned}
$$

with $\tau_{1}=(3 \beta+2 \gamma) /(\beta+\gamma)$. From Eqs. (5.29), it is straightforward to derive the leading singularities for cluster numbers, percolation order parameters, susceptibility, etc. Noting that in a cluster of size $n$, the fluctuation of the quantity $n_{s}=n_{A}^{c}-n_{B}^{c}$ should be of order $n^{1 / 2}(n>>1)$, Harris proposed ${ }^{16}$

$$
\begin{aligned}
W\left(n_{A}^{c}, n_{B}^{c}, p\right) & =W\left(n, n_{s}, p\right) \\
& =n^{-\tau_{1}} f_{1}\left[n\left(p-p_{c}\right)^{\beta+\gamma}\right]\left[\frac{1}{\sqrt{n}} \phi\left(\frac{n_{s}}{\sqrt{n}}\right)\right] .
\end{aligned}
$$

Furthermore,

$$
\sum_{n_{s}} W\left(n, n_{s}, p\right) B\left(n_{s}\right) \approx W(n, p) B\left(n^{1 / 2}\right) .
$$

Using Eqs. (5.30) and (5.31), we may roughly evaluate Eq. (2.15) for $\hat{\chi}_{t}$ at $T=0$ in the following way:

$$
\begin{aligned}
& \hat{\chi}_{t} / N=\sum_{n, n_{s}} W\left(n, n_{s}, p\right) \\
& \times\left[\frac{n_{s}^{2}}{\Gamma n}+\frac{1}{2 J} \sum_{i, j}\left[\epsilon_{i}-\frac{n_{s}}{n}\right]\right. \\
&\left.\times R_{i j}\left[\epsilon_{j}-\frac{n_{s}}{n}\right]\right] .
\end{aligned}
$$

The first term in the above sum is

$$
\begin{aligned}
\frac{1}{\Gamma} \sum_{n, n_{s}} W\left(n, n_{s}, p\right) \frac{n_{s}^{2}}{n} & \approx \frac{1}{\Gamma} \sum W(n, p) \frac{n}{n} \\
& \approx \frac{1}{\Gamma}\left(A_{1}+B_{1}\left|p-p_{c}\right|^{2-\alpha_{p}}\right) .
\end{aligned}
$$

Note that this expression is supported by our mean-field result, i.e., Eq. (5.12). The second sum in Eq. (5.32) can be roughly evaluated as

$$
\begin{aligned}
\sum_{n, n_{s}} W\left(n, n_{s}, p\right) \sum_{i, j} \epsilon_{i} \epsilon_{j} R_{i j} & \approx \sum_{n, n_{s}} W\left(n, n_{s}, p\right) \frac{n_{s}^{2}}{n^{2}} \sum_{i, j} R_{i j} \\
& \approx \sum_{n} W(n, p) \frac{1}{n} \sum_{i, j} R_{i j}
\end{aligned}
$$

If we allow for further decoupling, $\bar{n} \approx\left(p-p_{c}\right)^{-\beta-\gamma}$ and $^{19}$

$$
\sum_{i, j} R_{i j} \propto\left(p-p_{c}\right)^{-\phi-\gamma},
$$

which yields for the second sum a singularity of the form $\left(p-p_{c}\right)^{\beta-\phi}$. Putting together the leading singular terms, we find for $T=0$ susceptibility the following expression:

$$
\hat{\chi}_{t} \propto \frac{1}{\Gamma}\left(p-p_{c}\right)^{2-\alpha}+\frac{1}{J}\left(p-p_{c}\right)^{\beta-\phi} .
$$

Similar analyses can also be carried out for the finitetemperature expression of $\chi_{t}$, i.e., Eq. (2.21),

$$
\hat{\chi}_{t} / N=\sum_{n, n_{s}} W\left(n, n_{s}, p\right)\left[\frac{S^{2} n_{s}^{2}}{3 k_{B} T}+\frac{1}{J} \sum_{i, j} \epsilon_{i} \epsilon_{j} R_{i j}\right] .
$$

The second sum in Eq. (5.36) is similar to Eq. (5.34), while the first term gives

$$
\begin{aligned}
\frac{S^{2}}{3 k_{B} T} \sum_{n, n_{d}} W\left(n, n_{s}, p\right) n_{s}^{2} & \approx \frac{S^{2}}{3 k_{B} T} \sum_{n} W(n, p) n \\
& \approx \frac{S^{2}}{3 k_{B} T}\left(p-p_{c}\right)^{\beta}
\end{aligned}
$$

With this result, the finite-temperature susceptibility can be written as

$$
\hat{\chi}(T) \propto \frac{\left(p-p_{c}\right)^{\beta}}{T}+\frac{1}{J}\left(p-p_{c}\right)^{\beta-\phi} .
$$

These relations suggest a new scaling relation for $\tau$, namely,

$$
\tau=\phi-\beta \text {. }
$$

At the upper critical dimension $d_{c}, \tau=0$ as $\phi=\beta=1$, in agreement with our mean-field calculation. Since $\epsilon$ expansion results for $\phi$ and $\beta$ are available, ${ }^{18,19}$ we can quote the $\epsilon$-expansion prediction according to Eq. (5.39) to be

$$
\tau \approx \frac{2}{21} \epsilon
$$


The validity of these arguments and Eq. (5.40) can be established, however, only by a direct calculation of $\tau$. In $d=3$, the numerical calculations ${ }^{10}$ give $\tau \approx 0.5$, whereas according to Eq. (5.40), $\tau=0.3$.

The results contained in Eqs. (5.35) and (5.38) can be combined in elegant single scaling forms of the type

$\hat{\chi}_{t}=\frac{\left(p-p_{c}\right)^{\beta}}{k_{B} T} F_{1}\left[\frac{k_{B} T / J}{\left(p-p_{c}\right)^{\phi}}, \frac{\Gamma / k_{B} T}{\left(p-p_{c}\right)^{\beta+\gamma}}\right]$,

$\hat{\chi}_{t}=\frac{\left(p-p_{c}\right)^{2 \beta+\gamma}}{\Gamma} F_{2}\left(\frac{k_{B} T / J}{\left(p-p_{c}\right)^{\phi}}, \frac{\Gamma / J}{\left(p-p_{c}\right)^{\beta+\gamma+\phi}}\right)$.

Our finite-temperature result at zero anisotropy is covered by assuming

$$
F_{1}(x, 0)=A_{1}+B_{1} x, \quad x<1
$$

whereas the zero-temperature result at finite anisotropy follows on taking

$$
F_{2}(0, y)=A_{2}+B_{2} y, \quad y<1 .
$$

The scaling functions $F_{1}$ and $F_{2}$ are simply related by the equation

$$
F_{2}(x, x y)=y F_{1}(x, y)
$$

To summarize, we have presented a calculation of the zero-temperature perpendicular susceptibility of a diluted antiferromagnet. We have obtained analytically the critical behavior of this quantity in the mean-field approximation. We believe this to be a new result, which supports some earlier heuristic considerations. We also suggest a new scaling form for the perpendicular susceptibility and obtain a new scaling relation for the critical index $\tau$.

\section{ACKNOWLEDGMENT}

One of us (D.K.) is very grateful to Professor D. L. Huber for hospitality at the University of Wisconsin, numerous discussions, and some very valuable mathematical advice. The research was supported by the National Science Foundation, under Grant No. DMR-82-03704.

\section{APPENDIX A}

Expanding the product in Eq. (4.14), one finds that

$$
Z^{\prime}=s^{N} e^{N K z / 2} Z=\operatorname{Tr} \exp \left[s H \sum_{i} \epsilon_{i}\left(\delta_{\sigma_{i}, 1}-1\right)\right]\left[1+v \sum_{i, j} \delta_{\sigma_{i}, \sigma_{j}}+v^{2} \sum_{\langle i j\rangle,\langle k l\rangle} \delta_{\sigma_{i}, \sigma_{j}} \delta_{\sigma_{k}, \sigma_{l}}+\cdots\right] .
$$

One can associate each term of the expansion with an $N$-point graph in which a bond on neighboring sites $\langle i j\rangle$ denotes $v \delta_{\sigma_{i} \sigma_{j}}$ and its absence denotes 1 . Then the contribution to the graph can be written as a product over connected clusters, ${ }^{12}$ and

$$
Z^{\prime}=\sum v^{N_{l}(G)} \prod_{\begin{array}{c}
\text { product } \\
\text { over clusters } \\
\text { of } G
\end{array}} \text { (contribution of a cluster), }
$$

where $N_{l}(G)$ denotes the number of bonds in the graph $G$. Let $A_{n_{A}, n_{B}}(H)$ denote the contribution of a cluster containing $n_{A} A$ sites and $n_{B} B$ sites; then,

$$
Z^{\prime}=\sum_{G} v^{N_{l}(G)} \prod_{n_{A}, n_{B}}\left[A_{n_{A}, n_{B}}(H)\right]^{N W\left(G, n_{A}, n_{B}\right)},
$$

where $W\left(G, n_{A}, n_{B}\right)$ is the number of $n_{A}-n_{B}$ clusters in graph $G$ per site:

Thus,

$$
\begin{aligned}
A_{n_{A}, n_{B}}(H) & =\sum_{\sigma_{i}} \exp \left[s H \sum_{i} \epsilon_{i}\left(\delta_{\sigma_{i}, 1}-1\right)\right] \prod_{i} \delta_{\sigma_{i}=\sigma} \\
& =1+(s-1) e^{-s H\left(n_{A}-n_{B}\right)} .
\end{aligned}
$$

$$
Z=s^{-N} e^{-N K z / 2} \sum_{G} p^{N_{l}(G)}(1-p)^{N z / 2-N_{l}(G)} \prod_{n_{A}, n_{B}}\left[1+(s-1) e^{-s H\left(n_{A}-n_{B}\right)}\right] N W\left(n_{A}, n_{B}, G\right) .
$$

We now consider the free energy per site and take the $s \rightarrow 1$ limit in the following way:

$$
\begin{aligned}
f_{1} & =\lim _{N \rightarrow \infty} \lim _{s \rightarrow 1} \frac{1}{N(s-1)} \ln Z \\
& =\frac{z K}{2}-1+\sum_{G} p(G) \sum_{n_{A}, n_{B}} W\left(G, n_{A}, n_{B}\right) e^{-H\left(n_{A}-n_{B}\right)}
\end{aligned}
$$

where

$$
=\frac{z K}{2}-1+\sum_{n_{A}, n_{B}} \bar{W}\left(n_{A}, n_{B}\right) e^{-H\left(n_{A}-n_{B}\right)},
$$

$$
p(G)=p^{N_{l}(G)}(1-p)^{N z / 2-N_{l}(G)}
$$


is obviously the probability for the occurrence of a graph $G$, and $\bar{W}$ is the cluster number averaged over all graphs.

\section{APPENDIX B}

To do the mean-field theory, the following representation of the Potts Hamiltonian by $\mathrm{Zia}$ and Wallace ${ }^{20}$ proves very convenient:

$$
\beta \mathscr{H}=-(s-1) K \sum_{i, j} \mathbf{v}_{i} \cdot \mathbf{v}_{j}-(s-1) \sum_{i} \mathbf{v}_{i} \cdot \mathrm{e}^{1} H_{i},
$$

where $\mathbf{v}_{i}$ 's are $(s-1)$-dimensional vectors assuming $s$ -

discrete values given by $\mathrm{e}^{\alpha}$, where

$$
\mathrm{e}^{\alpha} \cdot \mathrm{e}^{\alpha}=1, \quad \mathrm{e}^{\alpha} \cdot \mathrm{e}^{\beta}=-(s-1)^{-1} .
$$

We take the field $H_{i}$ to be such, that

$$
H_{i}= \begin{cases}H_{A}, & i \in A \\ H_{B}, & i \in B\end{cases}
$$

The ordering occurs along $\mathrm{e}^{1}$, so that

$$
\begin{aligned}
& \left\langle\mathbf{v}_{i}\right\rangle=\psi_{A(B)} \mathrm{e}^{1}, \quad i \in A(i \in B) \\
& \psi_{A(B)}=\mathrm{e}^{1} \cdot\left\langle\mathbf{v}_{i}\right\rangle, \quad i \in A(i \in B) .
\end{aligned}
$$

The mean-field Hamiltonian can be written by the usual decoupling:

$$
\beta \mathscr{H}_{\mathrm{MF}}=-(s-1)\left[\sum_{i \in A}\left(z K \psi_{B}+H_{A}\right) \mathbf{v}_{i} \cdot \mathrm{e}^{1}+\sum_{i \in B}\left(z K \psi_{A}+H_{B}\right) \mathbf{v}_{i} \cdot \mathbf{e}^{1}-\frac{N z K}{2} \psi_{A} \psi_{B}\right] .
$$

The partition function can now be easily calculated to be

$$
\begin{aligned}
z_{\mathrm{MF}}= & \operatorname{Tr} e^{-\beta \mathscr{H}_{\mathrm{MF}}}=e^{-(s-1)\left(N_{z} K / 2\right) \psi_{A} \psi_{B}}\left[e^{(s-1)\left(H_{A}+z K \psi_{B}\right)}+(s-1) e^{-\left(z K \psi_{B}+H_{A}\right)}\right]^{N / 2} \\
& \times\left[e^{(s-1)\left(z K \psi_{A}+H_{B}\right)}+(s-1) e^{-\left(z K \psi_{A}+H_{B}\right)}\right]^{N / 2}, \\
f_{1}= & \left.\frac{1}{N(s-1)} \ln Z\right|_{N \rightarrow \infty, s \rightarrow 1} \\
= & \frac{z K}{2}\left(\psi_{A}+\psi_{B}-\psi_{A} \psi_{B}\right)+\frac{1}{2}\left(H_{A}+H_{B}\right)+\frac{1}{2}\left(e^{-\left(z K \psi_{B}+H_{A}\right)}+e^{-\left(z K \psi_{A}+H_{B}\right)}\right) .
\end{aligned}
$$

The equation for the order parameters $\psi_{A}$ and $\psi_{B}$, by minimizing $f_{1}$ with respect to $\psi_{A}$ and $\psi_{B}$, yields

$$
\psi_{A}=1-e^{-\left(z K \psi_{B}+H_{A}\right)}
$$

and

$$
\psi_{B}=1-e^{-\left(z K \psi_{A}+H_{B}\right)} .
$$

The correspondence of these equations with Eqs. (4.11) and $(4.12)$ is easily established if we set $\Gamma=0$,

$$
H_{A}=-H_{B}=\mathbf{h}_{\pi} \cdot \mathrm{W} \text {, and } \psi_{A(B)}=1-S_{A(B)}(\mathrm{W}) .
$$

\section{APPENDIX C}

We have

$$
\chi_{t}^{(1)}=+\int d^{n} W\left[\nabla_{W}\left[\frac{W^{\alpha} S_{0}^{(1)}(\mathbf{W})}{1+x S_{0}^{(1)}(\mathbf{W})}\right]\right]^{2}
$$

As before, we take the factor $\left[1+x S_{0}^{(1)}(\mathbf{W})\right]$ in the denominator to be a constant, and write

$$
\begin{aligned}
\chi_{t}^{(1)} & \lesssim \frac{1}{(1+x)^{2}} \int d^{n} W\left[S_{0}^{(1)}(\mathbf{W})^{2}+W_{\alpha}^{2} \nabla S_{0}^{(1)}(\mathbf{W}) \cdot \nabla S_{0}^{(1)}(\mathbf{W})+2 W_{\alpha} \frac{\partial S_{0}^{(1)}}{\delta W_{\alpha}}\right] \\
& \lesssim \frac{1}{(1+x)^{2}} \sum_{s, t} P_{s} P_{t} \int d^{n} W e^{-(s+t) \Gamma W^{2} / 2\left(1+s t \Gamma^{2} \mathbf{W}^{2} W_{\alpha}^{2}-2 s \Gamma W_{\alpha}^{2}\right)} \\
& \lesssim \frac{1}{(1+x)^{2}} \sum_{s, t} P_{s} P_{t}\left[\frac{2 \pi}{\Gamma(s+t)}\right]^{n / 2}\left|1+\frac{s t(n+2)}{(s+t)^{2}}-\frac{2 s}{s+t}\right| \\
& \lesssim \frac{1}{4} \sum_{s, t} p_{s} p_{t}\left[1+\frac{2 s t}{(s+t)^{2}}-\frac{2 s}{s+t}\right] .
\end{aligned}
$$

Noting that near $p_{c}$, for large $s,{ }^{15,16}$

$$
P_{s}=\frac{1}{s^{\tau_{1}-1}} f\left(s\left|r_{0}\right|^{\sigma}\right)+\text { nonsingular terms } .
$$

We can approximately determine these sums in meanfield theory, $\tau_{1}=\frac{5}{2}$ and $\sigma=2$. In evaluating the double sums, nonsingular contributions should also be kept, e.g.,

$$
\sum_{s, t} P_{s} P_{t}=\left\{\begin{array}{l}
\left(1-p_{\infty}\right)^{2} \approx 1-4\left|r_{0}\right|+O\left(r_{0}^{2}\right), \quad r_{0}<0 \\
1, \quad r_{0}>0
\end{array}\right.
$$

whereas the use of a scaling form will only give a contribution like $\left|r_{0}\right|^{2 \beta}=\left|r_{0}\right|^{2}$. Keeping this in mind one can easily estimate that all the sums in (C2) are nondivergent and simple functions of $\left|r_{0}\right|$. 
*Present address: Department of Physics, University of Roorkee, Roorkee, 247667, Uttar Pradesh, India.

${ }^{1}$ S. Kirkpatrick, in Ill-Condensed Matter, edited by R. Balian, R. Maynard, and G. Toulouse (North-Holland, Amsterdam, 1979', p. 323.

${ }^{2} \mathrm{An}$ up-to-date review of the state of the art can be found in Percolation Structure and Processes, edited by G. Deutscher, R. Zallen, and J. Adler (Annals of the Israeli Physical Society, Jerusalem, 1984), Vol. 5.

${ }^{3}$ A. Aharony, J. Stat. Phys. 34, 931 (1984).

${ }^{4}$ S. Alexander, in Percolation Structure and Processes, Ref. 2, p. 150.

${ }^{5}$ W. Brenig, P. Wolfle, and G. Dohler, Z. Phys. 246, 1 (1971).

${ }^{6}$ Y. Gefen, A. Aharony, and S. Alexander, Phys. Rev. Lett. 50, 77 (1983).

${ }^{7}$ S. Alexander and R. Orbach, J. Phys. (Paris) Lett. 43, L625 (1982).
${ }^{8}$ R. Rammal and G. Toulouse, J. Phys. (Paris) Lett. 44, L13 (1983).

${ }^{9}$ D. Kumar, Phys. Rev. B 30, 2961 (1984).

${ }^{10}$ A. B. Harris and S. Kirkpatrick, Phys. Rev. B 16, 542 (1977).

${ }^{11}$ D. J. Breed, K. Gilijamse, J. W. E. Sterkenberg, and A. R. Miedema, J. Appl. Phys. 41, 1267 (1970); Physica 68, 303 (1973).

${ }^{12}$ E. Lagendijk and W. J. Huiskamp, Physica 62, 444 (1972).

${ }^{13}$ M. J. Stephen, Phys. Rev. B 17, 4444 (1978).

${ }^{14}$ T. C. Lubensky, in Ill-Condensed Matter, Ref. 1, p. 405.

${ }^{15}$ M. J. Stephen, Phys. Rev. B 15, 5674 (1977).

${ }^{16}$ D. Stauffer, Phys. Rep. 54, 1 (1979).

${ }^{17}$ A. B. Harris (unpublished).

${ }^{18}$ A. B. Harris and T. C. Lubensky, J. Phys. A 17, L609 (1984).

${ }^{19}$ A. B. Harris, T. C. Lubensky, W. K. Holcomb, and C. Dasgupta, Phys. Rev. Lett. 35, 327 (1975).

${ }^{20}$ R. K. P. Zia and D. J. Wallace, J. Phys. A 8, 1495 (1975). 